\title{
Cytoplasmic particles in hepatocytes of patients with Australia antigen-positive liver disease
}

\author{
MARGARET E. HAYNES, DAVID W. G. HAYNES, AND ROGER WILLIAMS \\ From the Liver Unit, King's College Hospital, London
}

SYNOPSIS An electron microscope study was made of liver biopsy material obtained during life or shortly after death from 11 patients. In three of them, where serum was positive for Australia antigen (two had serum hepatitis and one active chronic hepatitis) the hepatocytes contained characteristic particles within membrane-bound cytoplasmic vesicles. The appearance of these particles was very similar to that of the Australia antigen particles found in the serum.

Two sizes of cytoplasmic particles were observed, with average diameters of about 26 and $46 \mathrm{~nm}$. Particles of both sizes often had a membrane-like outer component and a moderately electron-dense inner component. They differed in both size and structure from the mainly intranuclear particles described by previous authors and which were not found in the present patients. The nature of the vesicles containing the particles was not apparent.

It is now widely accepted that the presence in the blood both of characteristic particles and of an antigen known as the Australia antigen is indicative of infection by the serum hepatitis virus. The particles have been well described by Dane, Cameron, and Briggs (1970) among others and include two main types: $22 \mathrm{~nm}$ bodies, with little substructure and larger particles, about $42 \mathrm{~nm}$ in diameter, that have a dense outer coat about $7 \mathrm{~nm}$ across and an inner body of approximately $28 \mathrm{~nm}$. Since the specific antibody to Australia antigen will cause clumping of both these particle types (Almeida and Waterson, 1969), it is generally considered that the particles themselves constitute the antigen.

Australia antigen has also been identified in the faeces (Ferris, Kaldor, and Gust, 1970), the urine (Tripatzis and Horst, 1970), and synovial fluid (Onion, Crumpacker, and Gilliland, 1971) in patients with serum hepatitis. Only a few workers, however, have been able to demonstrate its presence in liver tissue. In 1970 Nowosławski, Brzosko, Madaliński, and Krawczyński, detected particles in nuclei of hepatocytes in six patients with lymphoproliferative disorders, all of whom were carriers of Australia antigen. These particles were spherical, about 18-25 $\mathrm{nm}$ in diameter, and had a dense outer coat and an apparently empty centre. Similar particles have been demonstrated by Nelson, Barker, and Danovitch (1970) in hepatocyte nuclei of a patient Received for publication 24 November 1971. with serum hepatitis, by Ahmed, Huang, and Spence (1971) in nuclei and cytoplasm of a patient with active chronic hepatitis, and by Huang (1971) in nuclei and cytoplasm of five patients with serum hepatitis or active chronic hepatitis. When present in the cytoplasm the particles were found singly or in small groups and were not within vesicles. Huang (1971) has also described particles of a rather different nature that were found in hepatocytes of one of his five cases: these particles were found in membranebound cytoplasmic vesicles, had a membrane-like outer component, and were approximately $35-40 \mathrm{~nm}$ in diameter.

In this paper we describe the finding in three patients of particles within vesicles in the cytoplasm of hepatocytes. The tissue was obtained by needle biopsy during life in two of the patients. Two patients had serum hepatitis, one active chronic hepatitis, and in all three the serum was positive for Australia antigen.

\section{Materials and Methods}

Material was examined from 11 patients in all and was obtained by aspiration needle biopsy during life or shortly after death. The clinical diagnoses were as follows: serum hepatitis (2), active chronic hepatitis (3), primary biliary cirrhosis (1), haemochromatosis (1), and acute hepatic necrosis (3), the last group including two patients in whom the condition 
followed halothane anaesthesia. The remaining biopsy was from the allograft at the completion of an orthotopic liver transplantation. Material obtained by needle biopsy during life was fixed within 15 seconds of removal. The postmortem biopsies were carried out within an hour of death, except in case 3 in which the interval was eight hours. The major part of each biopsy was fixed in formalin and used for light microscopy.

Material for electron microscopy was fixed for about three hours in $2 \%$ gluteraldehyde in $0.1 \mathrm{M}$ sodium cacodylate buffer, $p \mathbf{H} 7 \cdot 2$. Washing was in isotonic buffer, dehydration in graded alcohols, and propylene oxide, embedding in araldyte. Sections were cut using a Cambridge-Huxley microtome, stained with $2 \%$ uranyl magnesium acetate and lead citrate, and viewed with either a Siemens Elmiskop I or an AEI EM6B. Several blocks from each specimen were examined and two or three sections from each block: each section was normally examined for about one hour.

Sera were examined for the presence of Australia antigen and the corresponding antibody by standard techniques of immunodiffusion, electrophoresis, and complement fixation (World Health Organization Memorandum, 1970). Sera found to be positive were also examined by electron microscopy as follows: using an MSE Superspeed centrifuge, small volumes were given successive one-hour spins at $5000 \mathrm{~g}$, to remove debris, and at $25000 \mathrm{~g}$ to precipitate any particles present. The second pellet from each sample was washed and reprecipitated, then suspended in a few drops of isotonic saline, placed on a carbon-formvar coated grid and negatively stained in sodium phosphotungstate, pH 7.2.

\section{Results}

In three of the 11 patients, serum was found to be positive for Australia antigen or its antibody, and particles were observed in the liver cells on electron microscopy.

\section{CASE 1}

This was a 29-year-old male heroin addict, admitted after a 14-day history of jaundice. Liver biopsy was carried out five days after admission, at which time the serum titre of Australia antigen was $1 / 32$ by electrophoresis, $1 / 512$ by complement fixation. Light microscopy of the biopsy showed extensive collapse of the reticulin framework, with considerable portal tract inflammation and foci of necrotic hepatocytes.

On electron microscopy a considerable degree of cell damage was also apparent. About 2 to $3 \%$ of the hepatocytes were undergoing mitosis, while between 10 and $15 \%$ contained characteristic vacuoles $\frac{0}{\overrightarrow{5}}$ (Fig. 1). These vacuoles appeared to be bound by single membranes and contained groups of $\overrightarrow{\vec{F}}$ particles (Fig. 4b, c), mostly towards the periphery. Two sizes of particles were apparent, the smaller $\frac{}{0}$ type being approximately 15 times as numerous. The $\frac{\bar{c}}{\bar{s}}$ smaller particles had an average least diameter of $\bar{\Phi}$ $26.4 \mathrm{~nm}$, a range in diameters of 19 to $32 \mathrm{~nm}$; the 응 larger particles had an average least diameter of ${ }^{\infty}$ $45.7 \mathrm{~nm}$, a range of 39 to $68 \mathrm{~nm}$. Both types were $\overrightarrow{0}$ spherical and often had a densely stained membrane- $\overrightarrow{.}$ like outer component with a less electron-dense $\vec{\sigma}$ centre. The hepatocytes were normal in most other respects, with a high glycogen content and no apparent changes in nuclei and mitochondria. In or some cells the rough endoplasmic reticulum was swollen, but no abnormal inclusions were present in $\underset{\omega}{\stackrel{c}{c}}$ these swellings.

\section{CASE 2}

This 21-year-old woman had become jaundiced three months after a blood transfusion. When $\vec{c}$ admitted to hospital, after being ill for one week only, she was in deep hepatic coma, but she made a ${ }^{N}$ complete recovery after early and intensive treatment with heparin and fresh-frozen plasma (Rake, Shilkin, Flute, Lewis, Winch, and Williams, 1971). Liverō biopsy was performed on the 24th day, shortly before her discharge home. The blood was weakly $\stackrel{D}{\circ}$ positive at this time for antibody to Australia antigen $\overrightarrow{\vec{O}}$ and electron microscopy of the serum revealed the 3 presence of scattered antigen particles. Light microscopy of the biopsy showed a normal lobularo architecture with only slight septal formation, minimal infiltrate in the portal tracts, and a few areas? of spotty parenchymal necrosis.

Electron microscopy also indicated little cell damage. A few cells were undergoing necrosis, but $\frac{0}{3}$ no mitoses were seen. Of approximately 400 hepatocytes examined, a single cell showed a clearly ab-웅 normal appearance (Fig. 2). This cell contained several partially membrane-bound vesicles, similar to those described in case 1 , but tightly packed with an $N$ amorphous 'background' material that contained an large number of particles (Fig. 3). Particles can also be seen lying free in the cytoplasm, usually in close ${ }^{\omega}$ proximity to a vacuole. Almost all of the particles? are of the smaller type, with an average diameter of about $26 \mathrm{~nm}$ (Fig. 4d). The nucleus of this cell appeared to be normal, but several swollen and dis-rupted mitochondria were present and the endoplasmic reticulum was swollen.

CASE 3

This 34-year-old man had been found to have active chronic hepatitis six months before his final admis- 


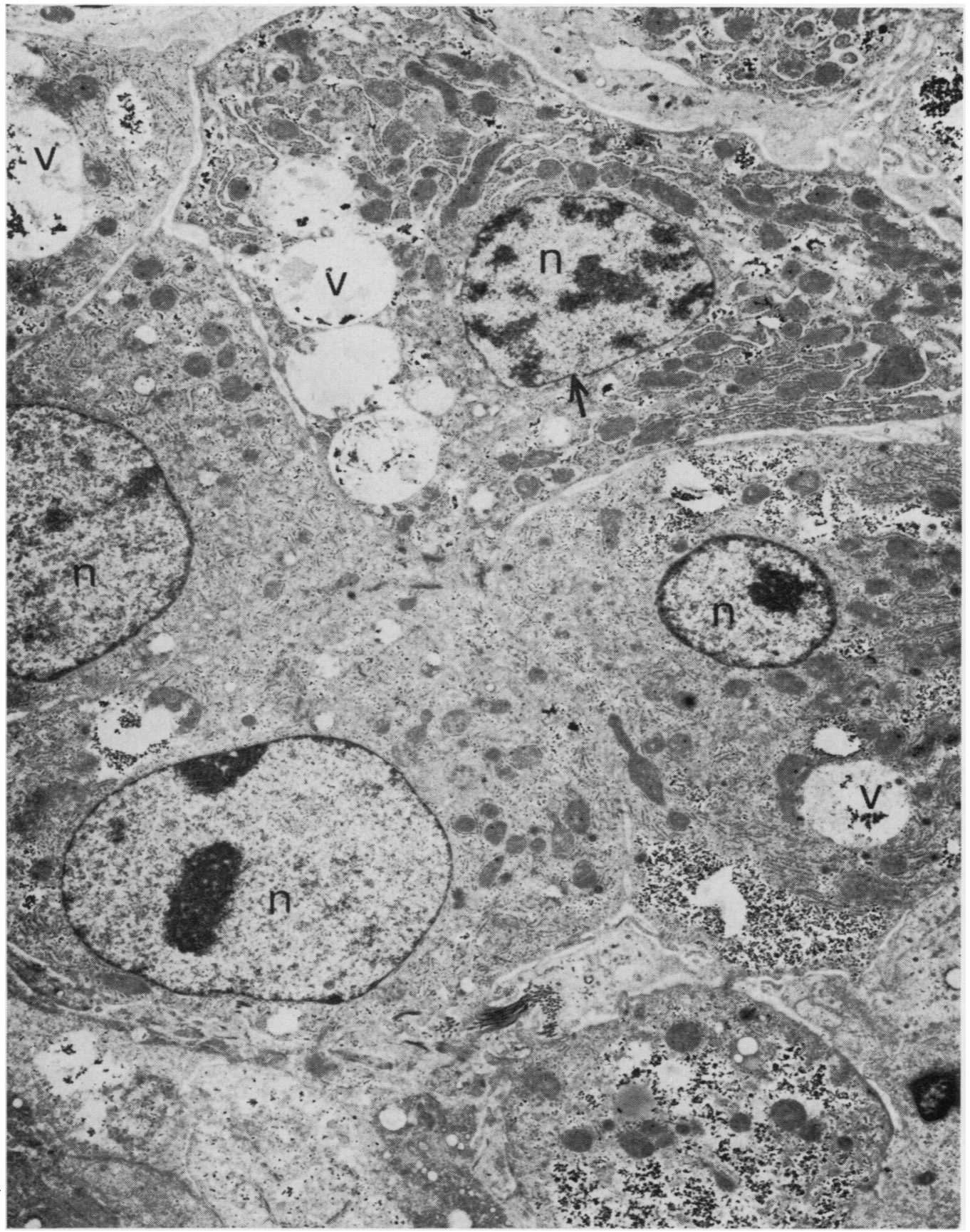

Fig. 1 Case 1. Hepatocytes containing membrane-bound vesicles, but otherwise of normal architecture. Arrowed nucleus contains chromosomes, as found shortly after mitosis. $\times 15000$.

$\mathrm{n}=$ nucleus, $\mathrm{v}=$ membrane-bound vesicle. 


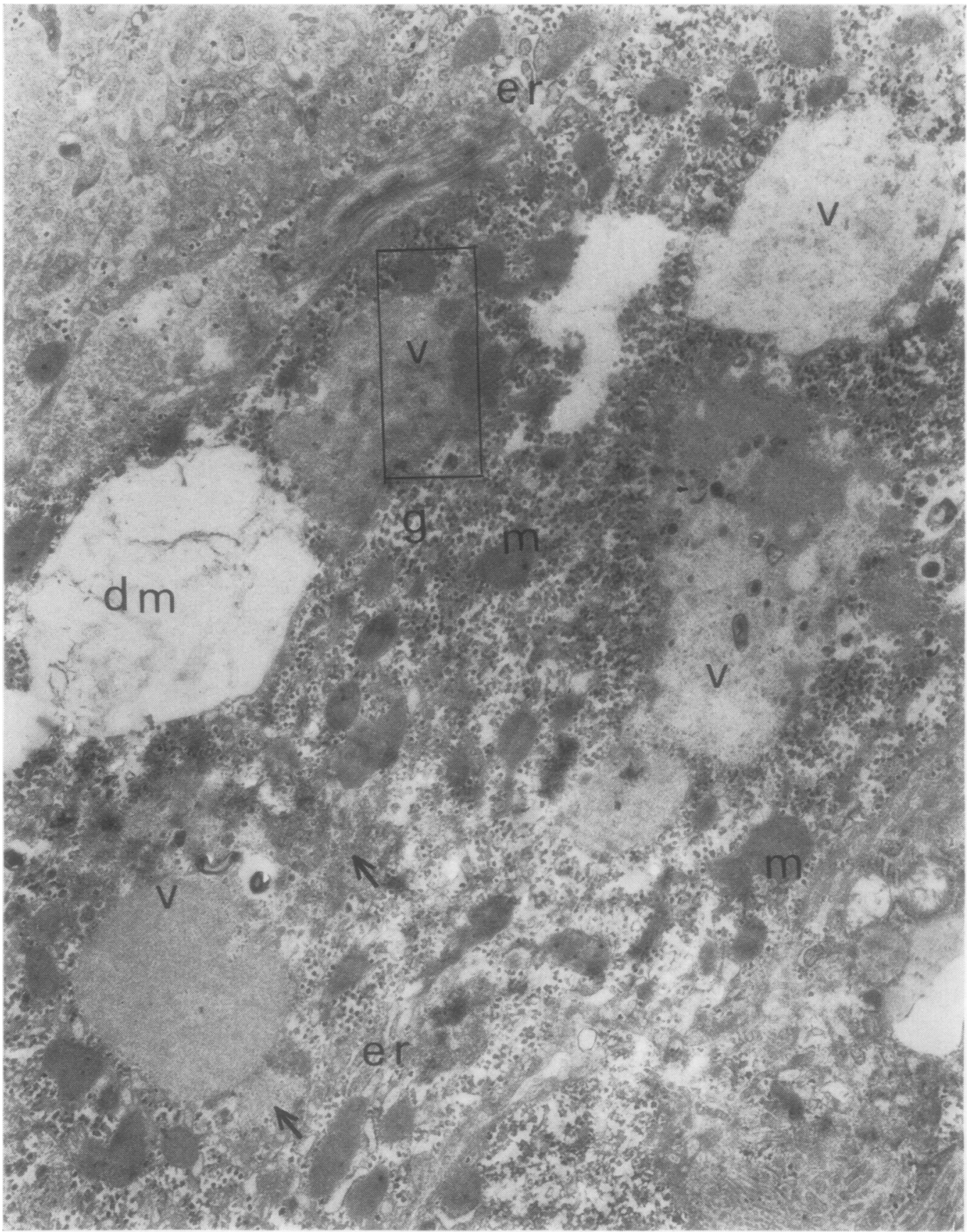

Fig. 2 Case 2. Hepatocyte showing large numbers of particles in membrane-bound vesicles or free in the cytoplasm (arrowed). A swollen and disrupted mitochondrion can also be seen. $\times 30000$. $\mathrm{g}=$ glycogen, $\mathrm{m}=$ mitochondrion, $\mathrm{v}=$ membrane-bound vesicle, $\mathrm{e} \mathrm{r}=$ endoplasmic reticulum, $\mathrm{d} \mathrm{m}=$ disrupted mitochondrion. 


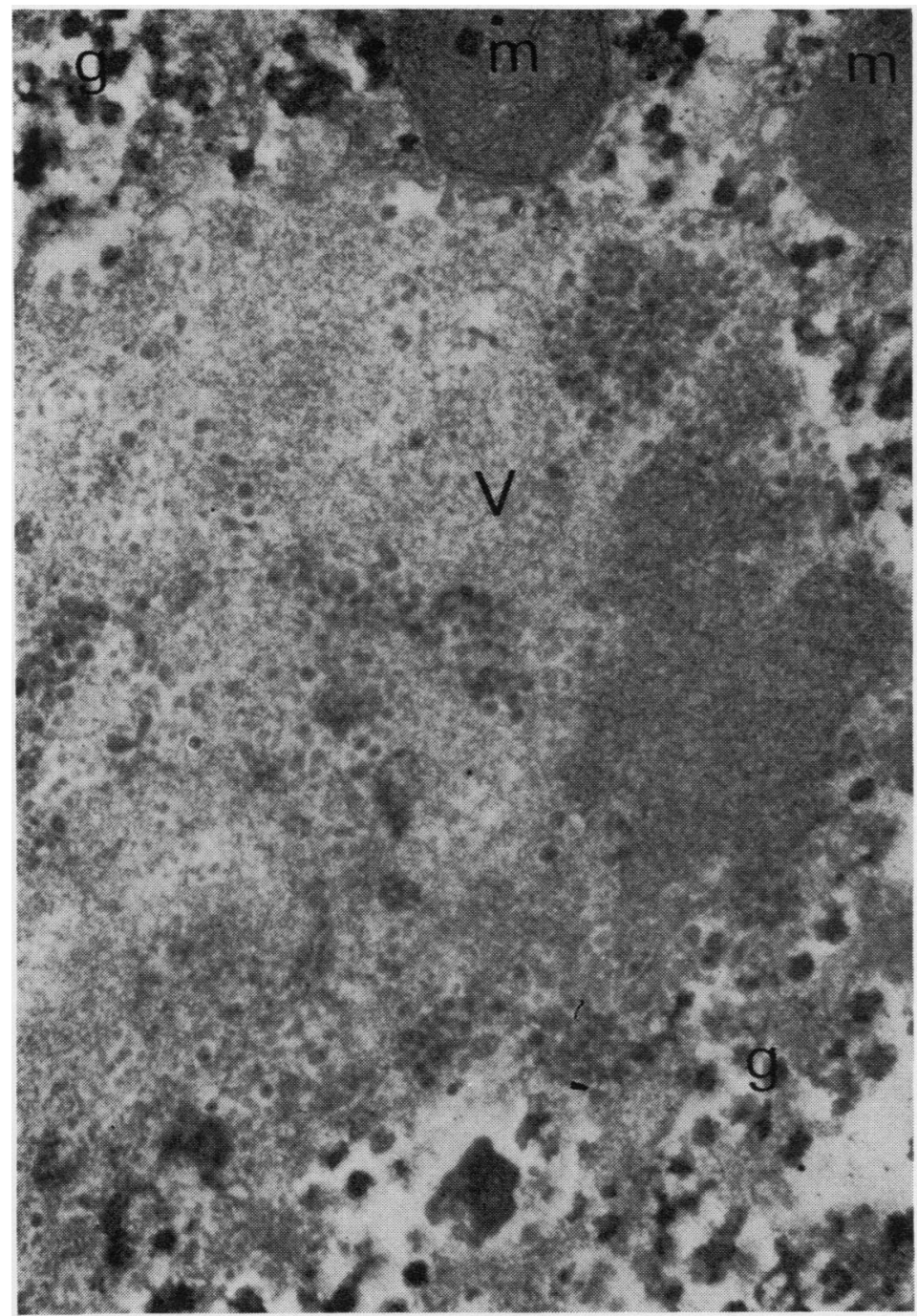

Fig. 3 Case 2. The encircled area of the hepatocyte shown in Figure 2. Numerous particles can be seen in an amorphous 'background' material. $\times 60000$.

$\mathrm{g}=$ glycogen, $\mathrm{m}=$ mitochondrion, $\mathrm{v}=$ membrane-bound vesicle.

sion to hospital with severe liver failure and bleeding oesophageal varices. Serum obtained shortly before death had a titre for Australia antigen of $1 / 16$ when examined by electrophoresis. At necropsy examination, the liver had the appearance of a macronodular cirrhosis and on light microscopy many of the nodules showed recent and extensive necrosis.

Electron microscopy of liver tissue taken by biopsy eight hours after death showed a very considerable degree of cellular damage, with some autolysis. Ten to $15 \%$ of the hepatocytes, the same proportion as in case 1 , contained membrane-bound vesicles. These vesicles were well packed with background material and contained large and small particles of the same size and appearance as in cases 1 and 2 (Fig. 4e). The ratio of small to large particles was similar to that found in case 1 .

Similar particles were demonstrated on electron microscopy of serum from these three cases (Fig. 4a). Large and small particles were always found, the average diameter of the smaller particles being $21 \cdot 1 \mathrm{~nm}$, with a range of 19 to $24 \mathrm{~nm}$, while the larger particles averaged $40.6 \mathrm{~nm}$ in diameter, with a range of 29 to $46 \mathrm{~nm}$. Very few large particles were found in serum from case 2 , while in the other two cases the ratio of small to large particles varied between 10:1 and 20:1 in different aliquots of serum. No substructure was apparent in these 


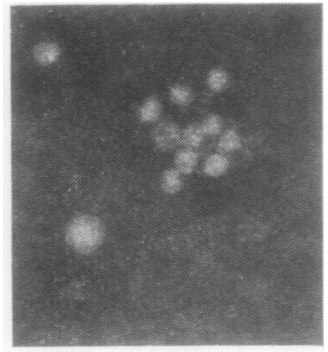

Fig. 4a.

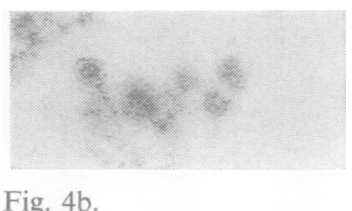

Fig. 4b.

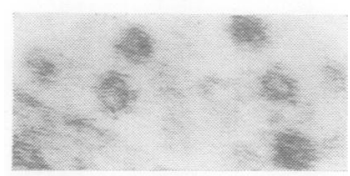

Fig. $4 c$.

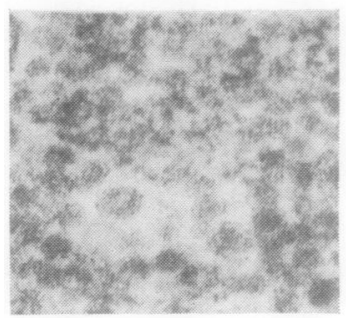

Fig. $4 d$.

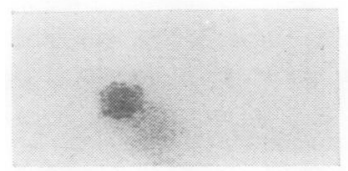

Fig. 4e.

particles, but in size and appearance they resemble the Australia antigen particles described by Dane $e t$ el (1970) and many other workers.

HISTOLOGICAL FINDINGS IN AUSTRALIAANTIGEN-NEGATIVE CASES

The biopsy material from these eight patients showed stained particles from sectioned material. Both large and small particles show a membrane-like 105000 . a-c Case 1, d Case 2, e Case 3.
Fig. 4b-e Positively outer component. $\times$
Fig. 4a Negatively stained particles from serum, showing large and small forms. $\times 120000$.
Fig. 4 High magnification

a number of hepatocellular abnormalities, including fibrotic changes, swollen endoplasmic reticulum, and disrupted mitochondria. However, in no case were there membrane-bound vesicles or particulate inclusions of the type described above.

\section{Discussion}

The particles demonstrated in the hepatocytes of the $\overrightarrow{0}$ three Australia-antigen-positive patients had a $\overrightarrow{\vec{H}}$ similar size and substructure in each case. They $\vec{\omega}$ differed in size and appearance from such normal cytoplasmic constituents as ribosomes and glycogen 0 granules. They resembled, in shape and in the of presence of two distinct sizes, the Australia antigen or particles found in serum from the same patients. The cytoplasmic particles were larger, with a diameter $5 \mathrm{~nm}$ greater on average, and showed a degree of substructure not apparent in the serum particles. However, in view of the considerable differences in methods of preparation and staining of liver tissue and material from serum, there seems no reason to suppose that the particles from these two sources ? were basically different.

It also seems likely that these particles are of the same nature as those demonstrated by Huang (1971) in hepatocytes of one out of five patients positive for Australia antigen. These particles were always found in the cytoplasm, principally within membranebound vesicles that were tightly packed with an amorphous 'background' material. They had a membrane-like outer component and a central area that was also fairly electron dense. The particles described by Huang were, however, of only one size, which is near to that of the larger particles found in the present patients.

The particles are, however, clearly different from those described by Ahmed et al (1971), Nelson et al (1970), and Nowosławski et al (1970), and which 음 Huang (1971) found in all five of his patients. These $\frac{D}{2}$ latter particles were found normally in the nucleus and when seen in the cytoplasm were not within $N$ vesicles. They were of only a single size between 18 and $26 \mathrm{~nm}$, and they had a dense, but not membranelike outer coat and often appeared to be hollow. $\omega$ Infected nuclei were described by Huang (1971) as being hypertrophied, with a considerably decreased $\varrho$ amount of chromatin, in contrast to the quite normal $\mathbb{D}$ appearance of the hepatocyte nuclei in the present $\stackrel{\mathscr{P}}{+}$ cases. It seems unlikely that the particles could have $\underline{T}$ been present in our material and have been masked $\stackrel{P}{P}$ by the amount of chromatin present as both Ahmed et al (1971) and Huang (1971) have shown them to be clearly visible in areas of normally dense chromatin.

A direct connexion between the two types of 
particle has yet to be established but it seems likely that the different types are related to different stages in the development of the infective agent of serum hepatitis. Huang (1971) suggests that the particles found in cytoplasmic vesicles represent the complete form of the serum hepatitis virus, that the central part of these particles is morphologically identical to the form found within the nucleus, and that the latter particles represent the initial stage of replication within an infected cell. However, it is our opinion that the central component of the particles described in the present material is more electron dense than the often apparently hollow central areas of the intranuclear particles. We also found particles with a membrane-like outer component that had a total diameter of only about $26 \mathrm{~nm}$, and a core about $15 \mathrm{~nm}$ in diameter: it is difficult to see how they could have been formed from the intranuclear type of particle.

Thus two different situations appear to have been described within the hepatocytes of patients with Australia-antigen-positive hepatitis. First there are the observations of a number of authors showing the apparent intranuclear replication of $22 \mathrm{~nm}$ particles with considerable disruption of the nucleus, sometimes also with some cytoplasmic replication and occasionally with the production, as demonstrated by Huang (1971), of rather different particles within cytoplasmic vesicles. Secondly there is the appearance described in the present patients, in whom intranuclear replication or damage was not apparent, but where there was an enormous production of particles within the cytoplasm of the larger type and a much greater degree of cytoplasmic damage. Whether these two situations represent different stages of a single process, or whether they are alternative manifestations of infection by the serum hepatitis virus, is uncertain, but it seems unlikely that either one could lead directly to the other. It is tempting to speculate that they are related to the immunological status of the infected patient, particularly as the material described by Nowosławski et al (1970), Huang (1971), and probably also Ahmed et al (1971) was obtained from patients either with diseases associated with a lack of immunological competence or were from patients on immunosuppressive therapy whereas only one of our three patients was receiving such treatment.

We have no information as to the nature of the cytoplasmic vesicles that contained the particles in our patients. A phagosomal origin seems the most likely, although lysosomes were not otherwise apparent in the infected cells. In case 2 degenerate mitochondria and swollen endoplasmic reticulum were particularly noticeable within the damaged cell, but in neither of these organelles could particles be demonstrated.

We are grateful to the King's College Hospital Voluntary Research Trust for their generous support and to Professor J. R. Garrett of the Electron Microscope Unit for his kind help.

\section{References}

Ahmed, M. N., Huang, S. N., and Spence, L. (1971). Australia antigen and hepatitis: an electron microscope study. Arch. Path., 92, 67-72.

Almeida, J. D., and Waterson, A. P. (1969). Immune complexes in hepatitis. Lancet, 2, 983-986.

Dane, D. S., Cameron, C. H., and Briggs, M. (1970). Virus-like particles in serum of patients with Australia antigen-associated hepatitis. Lancet, 1, 695-698.

Ferris, A. A., Kaldor, J., and Gust, I. D. (1970). Faecal antigen in viral hepatitis. Lancet, 2, 243-244.

Huang, S. N. (1971). Hepatitis-associated antigen hepatitis. Amer. J. Path., 64, 483-500.

Nelson, J. M., Barker, L. F., and Danovitch, S. H. (1970). Intranuclear aggregates in the liver of a patient with serum hepatitis. (Letter.) Lancet, 2, 773-774.

Nowoslsawki, A., Brzosko, W. J., Madalikski, K., and Krawczykski, K. (1970). Cellular localisation of Australia antigen in the liver of patients with lymphoproliferative disorders. Lancet, 1, 494-498.

Onion, D. K., Crumpacker, C. S., and Gilliland, B. C. (1971). Arthritis of hepatitis associated with Australia antigen. Ann. intern. Med., 75, 29-33.

Rake, M. O., Shilkin, K. B., Flute, P. T., Lewis, M. L., Winch, J., and Roger Williams (1972). Early and intensive therapy of intravascular coagulation in acute liver failure. (In preparation.)

Tripatzis, I., and Horst, H. G. (1971). Detection of Australia-SHantigen in urine. Nature (Lond.), 231, 266-267.

World Health Organization Memorandum (1970). Viral hepa titis and tests for the Australia (hepatitis-associated) antigen and antibody. Bull. Wld Hlth Org., 42, 957-992. 